\title{
ERRATA TO \\ GENERALIZATIONS OF THE PARTIAL, PART AND BIPARTIAL CANONICAL CORRELATION ANALYSIS
}

\author{
SiK- Yum LeE
}

THE CHINESE UNIVERSITY OF HONG KONG

Several corrections must be made in Lee [1978]. They are as follows:

(i) page 428 , line 15 from the top, the expression for $\Sigma_{12}^{*}$ should read

$$
\Sigma_{12}^{*}=\Sigma_{12}-\Sigma_{13} \Sigma_{33}^{-1} \Sigma_{32}-\Sigma_{14} \Sigma-1 \Sigma_{42}+\Sigma_{18} \Sigma_{33}^{-1} \Sigma_{34} \Sigma_{44}^{-1} \Sigma_{42}
$$

(ii) page 429 , line 5 from the top should read

$$
\Sigma_{11.94}=\Sigma_{11.8}-\Sigma_{14.8} \Sigma_{4.1}^{-1} \Sigma_{41.3} \text {. }
$$

(iii) page 429 , line 16 from the top, replace $\tilde{r}_{t(1.34)^{2}(2.38)}^{2}(2 . s s)$ by

$$
r_{i(1.94)(2.38)}
$$

(iv) page 429 , line 13 from the bottom should read

$$
e_{1.34}=e_{1.8}-\Sigma_{14.8} \Sigma_{44.8}^{-1} e_{4.3} .
$$

(v) page 429 , line 2 from the bottom should read

$$
\Sigma_{12}^{*}=\Sigma_{12.3}-\Sigma_{14.3} \Sigma_{44.8}^{-1 .} \Sigma_{42.3}-\Sigma_{18.9} \Sigma_{-5.3}^{-1} \Sigma_{52.3}
$$

\section{REFERENCE}

Lee, Sik-Yum. Generalizations of the partial, part and bipartial canonical correlation analysis. Psychometrika, 1978, 44, 427-431. 